\title{
Enhancing Visual Diagnosis of X-Ray Images by the Method of Selective Dilation
}

\author{
Sachdeep.S \\ VIT University \\ School of Electrical Engineering \\ Vellore, Tamilnadu, India
}

\author{
Monica Subashini.M \\ VIT University \\ School of Electrical Engineering \\ Vellore, Tamilnadu, India
}

\begin{abstract}
This paper presents a basic design and experimental results of a crack detection method on X-Ray images. The proposed method utilizes a customized algorithm wherein a section of the X-Ray image suspected to contain the irregularity, like a crack in the bone, is separated from the rest of the X-Ray image and the cropped image is altered so as to make the presence of irregularities more easily detectable. This primarily involves the detection and expansion of the irregularity in terms of size, or the number of pixels it occupies in the image. The algorithm helps isolate the irregularity and selectively dilate it, without interfering with the other parts of the image, thereby making it more easily visible to the human eye. This operation is accomplished by a Matlab supported operation called dilation. In a grayscale XRay image, the pixels constituting the image may take several intensity values. This obscures the distinction between boundaries, impeding visual diagnosis. This problem is avoided by converting the grayscale image to a binary image, creating a clear distinction between boundaries. This paper investigates the possibility of employing this approach to provide detection and selective amplification of irregularities given the region in the image in which the irregularity is suspected to lie. Then the processed image is compared with the original input image. The application of the proposed method for enhancing visual diagnosis is demonstrated by examples.
\end{abstract}

\section{General Terms}

Algorithm , Image segmentation

\section{Keywords}

Erosion, Matlab, Selective Dilation, X-Ray Images

\section{INTRODUCTION}

An X-ray image or Radiograph is obtained by placing a part of the patient in front of an X-ray detector and then illuminating it with a short X-ray pulse. Bones contain much calcium, which due to its relatively high atomic number absorbs $x$-rays efficiently. This reduces the amount of $\mathrm{X}$-rays reaching the detector in the shadow of the bones, making them clearly visible on the radiograph. Radiographs are useful in the detection of pathology of the skeletal system as well as for detecting some disease processes in soft tissue. Many researches are going on with X-Ray images on different applications referred from [1] and [2]. There are more recent attempts to detect cracks by image segmentation referred from [3] and [4]. A fracture results in producing an isolated region not completely filled with calcium and thus partially permeable to X-Rays, characterized by black regions on the radiograph. Traditional plain X-rays are less useful in the imaging of soft tissues such as muscles.[5] The primary application of X-Ray images is for detecting fractures or cracks in the bone acquired through injury. Sometimes, a crack in the bone may be so small that it is virtually impossible to detect its presence through observation of the X-Ray radiograph. Treatment for cracked bones involves immobilization of the affected region with a plaster or fiberglass cast or splint which holds the bones in position of until the crack heals on its own [6]. However, such treatment is harmful when administered in the absence of a crack, as it may lead to edema or swelling due to accumulation of fluid and may also restrict the flow of blood to the bones [7].

Thus, it is vital to know whether or not a crack is present and also its location to the precision of a few millimeters so that the appropriate treatment may be administered in time. In such cases, the proposed method may be applied to make the crack visible by expanding its dimensions. Image wide dilation distorts the entire image, making it more difficult to detect the presence and location of cracks. Thus, the narrow region where the crack is suspected to lie in should be dilated selectively so as to make the crack visible to the observer. The method of selective dilation can locate the cracks when they are too small to be seen, or confirm their absence, which is the purpose of obtaining a radiograph.

\section{SELECTIVE DILATION}

The method of selective dilation, as the name suggests, is used to provide selective image amplification to the irregularities, like cracks, on an X-Ray image to help in their visual detection.

\subsection{Algorithm}

The algorithm developed for the proposed method is described in the below subsections.

\subsubsection{Conversion to binary image}

In an X-Ray radiograph, the pixels constituting the image may take several intensity values, corresponding to the level of radiation that reaches the detector. This obscures the distinction between boundaries, making direct visual diagnosis a more difficult task. Quantizing the pixel intensity values to either 0 or 1 greatly reduces the problems involved by creating a clear distinction between boundaries. Besides, the image pixels may now be easily manipulated as the image may be thought as a large matrix of size $[\mathrm{m}, \mathrm{n}]$ with each of its pixels assuming intensity values of either 0 or 1 , 
corresponding to black or white respectively. White represents the bones while the black represents either cracks or fleshy regions through which $\mathrm{X}$-Rays can pass. This quantization to binary levels is accomplished by the use of the im2bw function, which yields a quantized binary image corresponding to the grayscale X-Ray image.

The read image is first converted to black and white using the rgb2gray function. The graythresh function computes a global threshold that can be used to convert a multiple intensity image to a binary image with im $2 \mathrm{bw}$. It returns a normalized intensity threshold value that lies in the range $[0,1]$, depending on the intensity distribution of the pixels lying on the image. The graythresh function uses Otsu's method, which chooses the threshold to minimize the intraclass variance of the black and white pixels. The command graythresh returns the effectiveness metric, EM, as the second output argument corresponding to the black and white Xray image specified as a parameter. The effectiveness metric is a value in the range $\left[\begin{array}{ll}0 & 1\end{array}\right]$ that indicates the effectiveness of the thresholding of the input image. The lower bound is attainable only by images having a single gray level, and the upper bound is attainable only by two-valued images.

The im 2 bw function converts the grayscale image to a binary image. The output image replaces all pixels in the input image with luminance greater than the value returned by the graythresh function with the value 1 (white) and replaces all other pixels with the value 0 (black). This process of conversion to grayscale image lowers the variation in pixel intensity values, creating a better distinction at boundaries. This makes it easier for doctors to visually diagnose irregularities like cracks.

The threshold value $\mathrm{L}$ lies in the range [0,1], regardless of the class of the input image. Without the use of the function "graythresh" to compute the value of the threshold pixel intensity, the argument automatically specifies the value and the threshold pixel intensity is assigned the value 0.5. After considering the values of pixel intensities and comparing with the computed threshold value, each pixel is assigned new quantized intensity values by the im 2 bw function and the indexed image I of continuous pixel values is converted to a binary image, which is better suited for image manipulation.

\subsubsection{Cropping}

As the name suggests, selective dilation is based on the principle of dilating a narrow region of interest specified by pixel co-ordinates. Dilating the entire image distorts the view completely and makes detecting cracks even more difficult than it already is. The region of interest is specified keeping in mind the possible location of injury and the point of origin of pain. This rectangular region of interest is extracted from the image and assigned a new name by the imcrop function referred from [8] .The rectangular crop region for the imcrop function has been determined by observing the region of interest and specifying the upper and lower $\mathrm{X}$ and $\mathrm{Y}$ coordinates of the image. This cropped image is stored as a binary matrix and is displayed as a figure. Cropping out the region of interest has narrowed down the region of operation to the region of interest in the image.

\subsubsection{Selective Dilation}

Now that attention is narrowed down to the location where the irregularity may be located, only this portion of the image can be dilated to check for its presence. However, the imdilate function considers the object of interest to be white in colour, set against a black background and using the function results in expansion of the white regions of bone, further obscuring the irregularity.

A method is needed which will result in the expansion of the black regions of the irregularity rather than the white regions of bone. This is accomplished specifying the imerode function, which erodes the white regions of bone, resulting in increased area for the black region of irregularity. In order to erode, the structural element is first specified as disk with a depth of 9 pixels using the strel function, which returns a structural element given the type of erosion that is to be used and the required pixel depth of the affected region. Specifying the pixel depth of 9 correspondingly increases the length and width of the irregularity by at least 9 pixels, adding to its visibility.

This is particularly useful when the dimensions of the irregularity of interest are so low as to escape detection. The process of dilating only the regions surrounding the crack performs the same function as a amplifier when used to increase the strength of a signal too weak to be measured. Thus the method of selective dilation is a method of amplification of irregularities to aid in their visual detection.

\subsubsection{Re-insertion of the dilated region of interest into the original image}

The cropped image is scaled up in display and thus, the view of the dilated cropped image does not give one an idea of the actual dilated dimensions of the crack. In order to aid an effective visual diagnosis, the original image must be modified and the region surrounding the possible irregularity must be replaced by dilated image of the region around the crack. This would give an observer an idea of the actual dimensions of the dilated irregularity, which he can estimate by comparing with the size of other structures present in the original image. This requires the superimposing of the cropped region in the original image with the dilated version of the cropped region. As MATLAB does not provide a predefined function for superimposing an image on another, this operation must be accomplished by assigning the values of pixel intensities. The pixels in the region surrounding the crack in the original image are assigned the binary intensity values of the corresponding pixels present in the dilated version of that region.

This operation is accomplished by means of a nested for loop, which traverses the entire area of the cropped region in the original image, replacing original pixel intensity values with the corresponding values obtained from the dilated version of the cropped region. The loop variables $i$ and $j$ are set the upper limits as the dimensions of the cropped region. The pixels in the original image are accessed by adding the values of the minimum $\mathrm{x}$ and $\mathrm{y}$ co-ordinates of the cropped image to the loop variables that represent traversal along the $\mathrm{x}$ and $\mathrm{y}$ axes respectively. By assigning the pixels of the original image the intensities of the corresponding pixels in the dilated image, the region surrounding the crack has been effectively replaced with the dilated version of the region. This makes it easier to diagnose the presence of irregularities, especially when the dimensions of the suspected irregularity are so low as to escape visual detection. 


\subsection{Steps followed in implementing selective dilation}

MATLAB is a high-performance language for technical computing and interactive environment for algorithm development [9].

Step 1: The image is read using the 'imread' function, which reads a grayscale or color image from the file specified by the filename and stores in the rgb matrix I.

Step 2: The image I may contain a bluish tinge due to the nature of the X-Ray film. It is fed to the 'rgb2gray' function to return its grayscale form GI.

Step 3: The grayscale image GI has multiple pixel intensity values corresponding to various levels of brightness, the 'im2bw' function quantizes these to binary levels by comparing the intensities with a threshold yielding a quantized binary image BW corresponding to the grayscale image GI.

Step 4: Using the binary image BW and the co-ordinates specifying the region of interest, the 'imcrop' function crops out the region of interest from BW, returning a cropped binary image $\mathrm{J}$, representing the region where the irregularity is supposed to lie.

Step 5: The cropped binary image $\mathrm{J}$ is subjected to dilation using the imerode function. As the irregularity is black in colour, it is dilated by eroding away the surrounding white regions using the imerode function, after specifying the type of erosion and the required pixel depth, returning the dilated image JD.

Step 6: The dilated image JD is superimposed on the complete binary image BW on the region from where image $J$ is cropped out. This operation is accomplished by assigning the values the binary pixel intensity values in the region surrounding the crack to the values of intensities of corresponding pixels present in the dilated version of that region by means of a nested for loop traversing the entire area of the cropped region in the original image using location of ROI data. The limits of the loop variables are set considering the upper co-ordinate limits (UVx,UVy) and the lower coordinate limits (LVx, LVy). This step returns the resultant image $\mathrm{K}$ produced by superimposing image JD on the region of interest.

Step 7: The images BW, J, JD and K are displayed and the results are observed.

Researchers have worked on similar lines with 3 dimensional images as referred from [10] and [11].

\subsection{Flowchart}

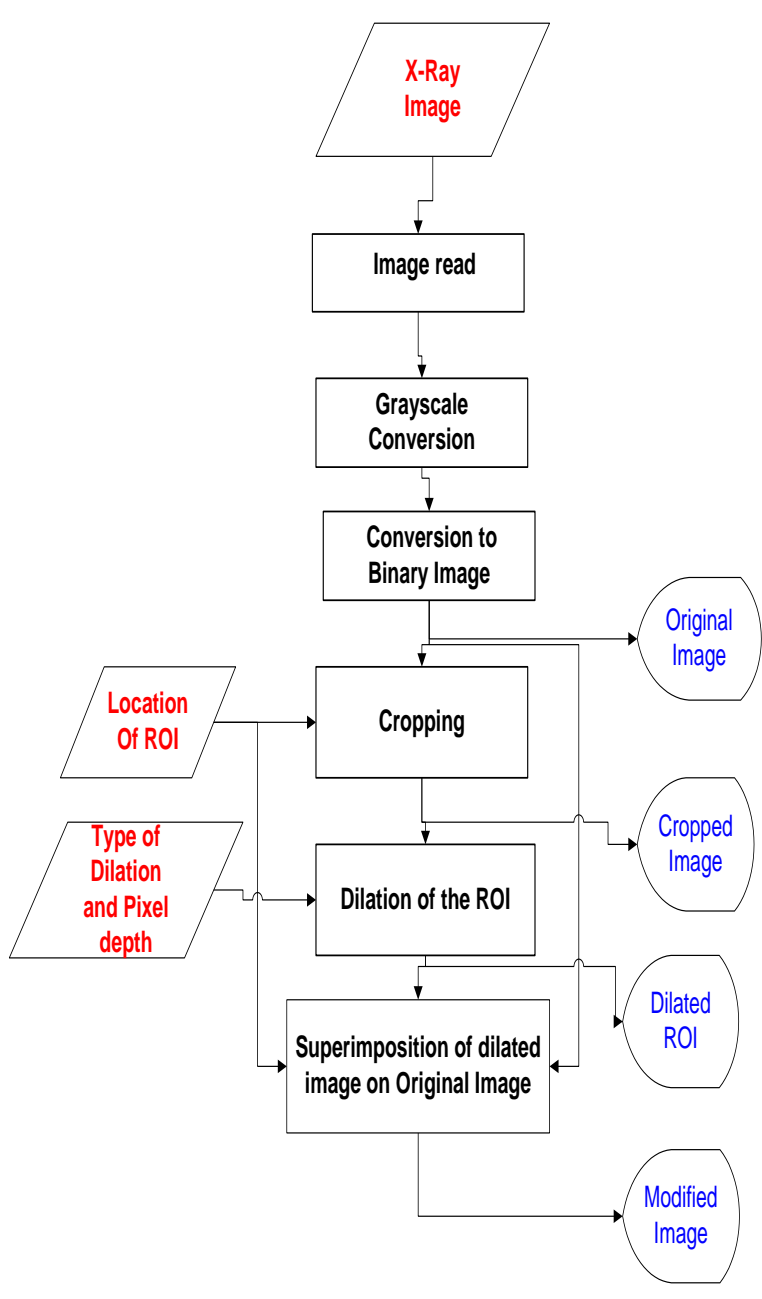

Figure 1: Flowchart for Selective Dilation

\subsection{Procedure followed and results obtained}

The X-ray images dataset was downloaded from [12]. Those images were chosen which showed the presence of a small crack whose visibility was improved significantly using the method of selective dilation. The effectiveness of this method can be better demonstrated with images with smaller cracks which cannot be spotted initially. Such images could not be found even after repeated searches on various X-Ray image databases.

The proposed method is used to enlarge a crack on an X-Ray of a pelvis shown in Figure 2 using the method of selective dilation. The scanned X-Ray image is of size $156 * 127$ pixels as shown in Figure 2. By observing the location of the crack, the region of interest may be narrowed down to a rectangle whose end points are $(63,38),(81,38),(63,56)$ and $(81,56)$. Ideally, the crack is too small to be seen and the region of interest should be selected on the basis of the possible location of injury and the possible source of pain. The rectangular region of size $18 * 18$ pixels is cropped and dilated. The dilated image is re-inserted into the original image in order for the observer to get an idea about the size of the dilated crack and to aid the observer in determining the location of the crack. It should be noted that the Y co-ordinate is 1 at the top of the 
image and increases with downward traversal. In terms of the image matrix, the $\mathrm{Y}$ co-ordinate of a particular pixel is the line number of the horizontal binary sequence in which the pixel is located and the $\mathrm{X}$ co-ordinate is the line number of the vertical binary sequence in which the pixel is located.

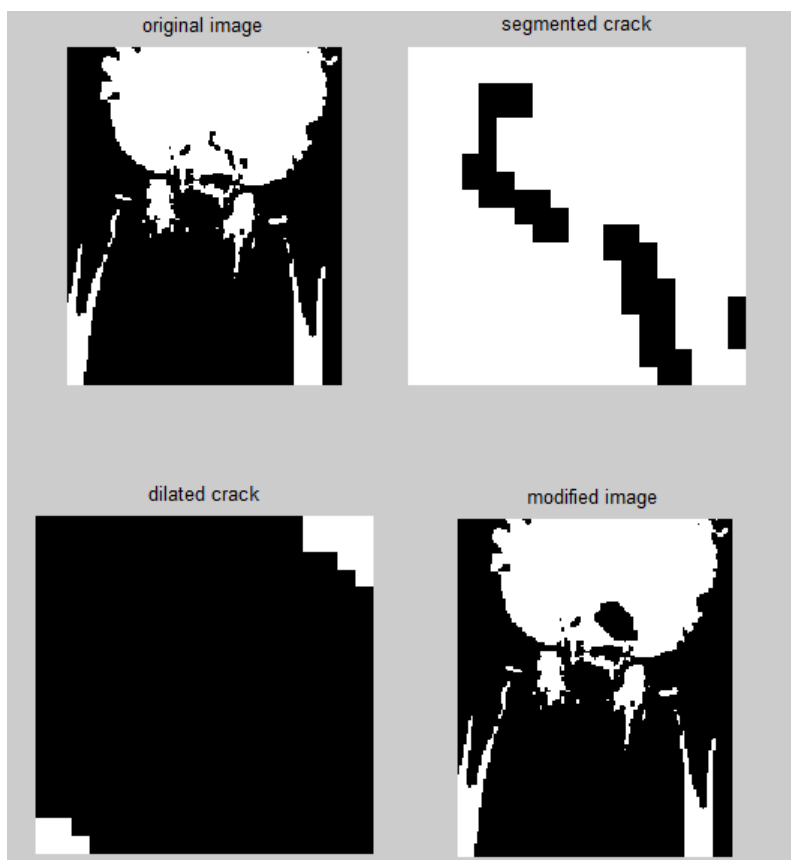

Figure 2: Pelvis X-Ray Image

Similarly the process is repeated for some images which are shown in Figures 3,4 and 5.

In Figure 3, the scanned X Ray image is of size $170 * 126$ pixels and the region of interest which is a rectangle that lies within the pixel co-ordinates $(45,1),(55,1),(45,30)$ and $(55,30)$.

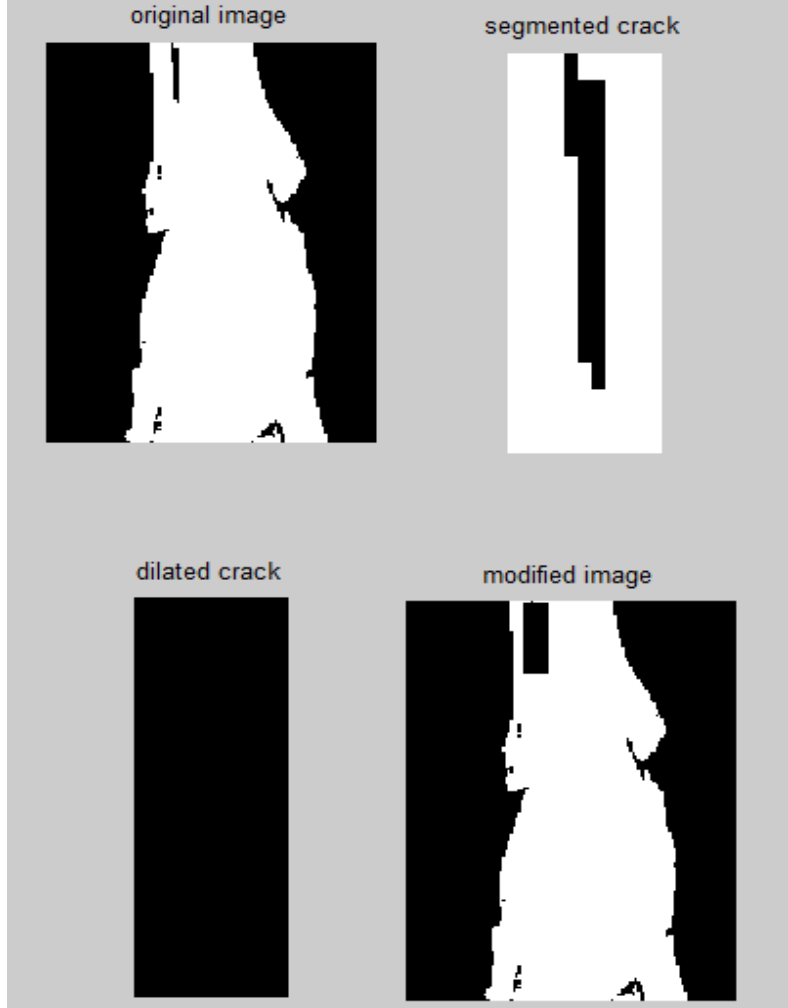

Figure 3: Ankle X-Ray Image

The size of image shown in Figure. 4 is $153 * 127$ pixels and region of interest is the rectangle that lies within the pixel coordinates $(104,60),(109,60),(104,80)$ and $(109,80)$

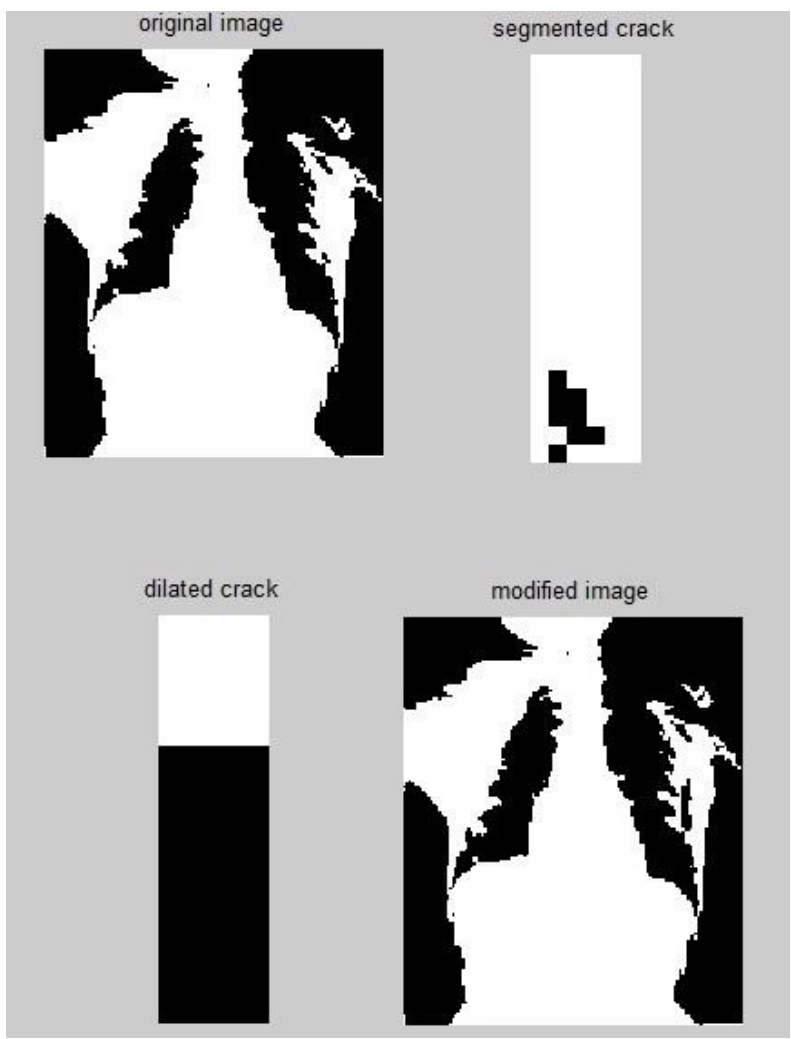

Figure 4: Chest X-Ray Image 
The scanned $x$ ray image shown in Figure. 5 is of size $90 * 127$ pixels. Region of interest is the rectangle that lies within the pixel co-ordinates $(83,46),(92,46),(83,50)$, and $(92,50)$

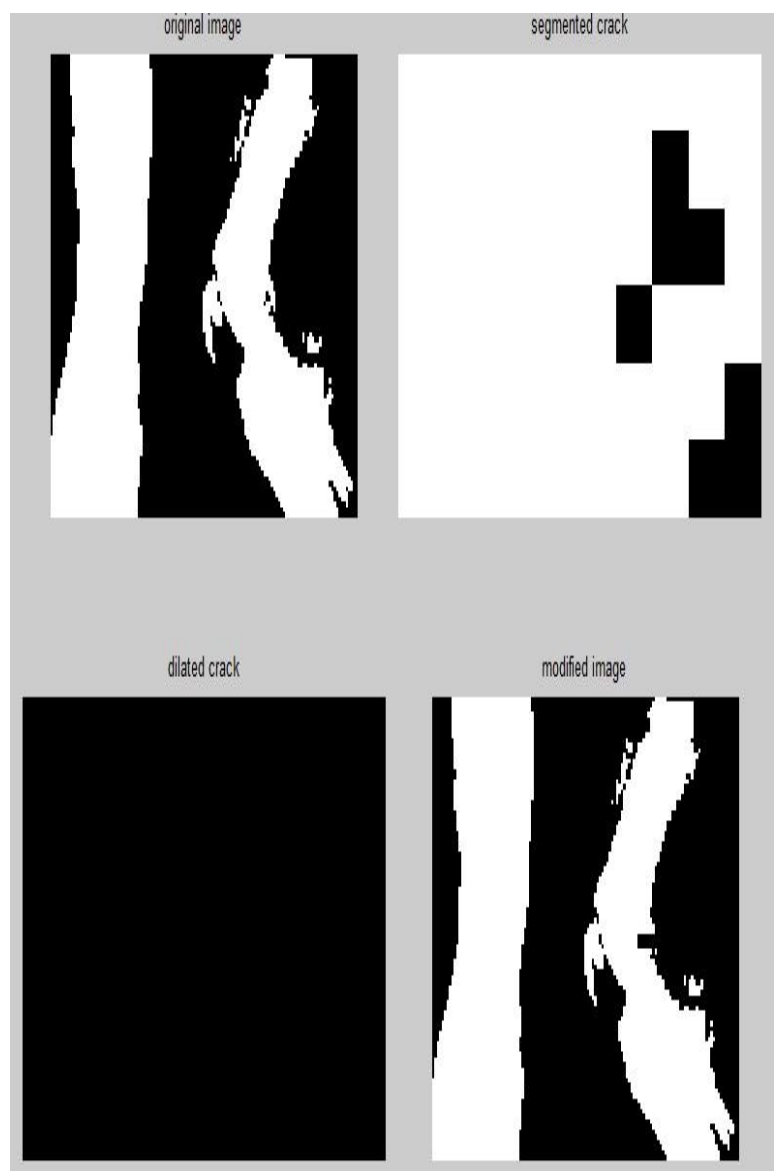

Figure 5: Exostoses X-Ray Image

\section{CONCLUSION}

An alternative to the processing of an image by the method of selective dilation is the use of predefined MATLAB filters, like Wiener filter, Lucy-Richardson filter, Imfilter or the ROI filter. These predefined MATLAB image filters merely deblur an image on the whole by either extracting signal noise or by assigning modified pixel intensity values taking into consideration the intensities of the surrounding pixels.

However these filters do not help in isolating the irregularity of interest. They also do not make use of the data available from the patient's report, with which this method uses to specify the region of interest keeping in mind the possible location of injury and the point of origin of pain. The ROI filter, despite narrowing down the filtering to the region of interest, merely performs deblurring of a specific region without selectively modifying the irregularity of interest. On the whole, these filters provide a clearer image which may or may not enhance the capability of an observer to make a visual diagnosis. However, the method of selective dilation assures us of a result confirming either the presence or absence of an irregularity. In this regard, the processing of an image by the method of selective dilation is found to be superior to the use of predefined MATLAB filters on the basis of the observed results.

\section{ACKNOWLEDGEMENTS}

This work has been supported by School of Electrical Engineering, VIT University.

\section{REFERENCES}

[1] Shiying Hu, Eric A. Hoffman and Joseph M. Reinhardt, "Automatic Lung Segmentation for Accurate Quantitation of Volumetric X-Ray CT Images", IEEE Transactions on Medical Imaging, vol. 20, no. 6, june 2001.

[2] J. Duryea and J.M. Boone, "A fully automated algorithm for the segmentation of lung fields on digital chest radiographic images", Med Phys., vol. 22, no. 2, pp. 183191, 1995.

[3] Sachin R.Mahajan, P.H.Zope and S.R.Suralkar, "Review of An Enhance Fracture Detection Algorithm Design Using X-Rays Image Processing", International Journal of Innovative Research in Science, Engineering and Technology, Vol. 1, Issue 2, December 2012.

[4] Jie Wu, Pavani Davuluri, Kevin R. Ward, Charles Cockrell, Rosalyn Hobson and Kayvan Najarian, "Fracture Detection in Traumatic Pelvic CT Images", International Journal of Biomedical Imaging, Volume 2012, 2012

[5] http://en.wikipedia.org/wiki/X-ray\#Radiographs"/ 'Information about X-Ray radiographs'

[6] http://www.medicinenet.com/fracture/page11.htm\#what_ is_the_treatment_of_a_bone_fracture/'Information on fracture treatment,

[7] http://www.ncbi.nlm.nih.gov/pmc/articles/PMC2950182/ 'Use of splints to treat fractures'

[8] M.S. Brow, "Method for Segmentation Chest CT Image Data Using an Anatomical Model: Preliminary Results," IEEE Transactions on Medical Imaging, vol. 16, no. 6, pp. 828-839, 1997.

[9] http://www.mathworks.in/'Syntax for functions used'

[10] Y. Kang, K. Engelke and W. Kalender, "A new accurate and precise 3-D segmentation method for skeletal structures in volumetric CT data", IEEE transactions on medical imaging, vol. 22, no. 5, pp. 586-98, May 2003.

[11] X. Zang, Y. Wang, J. Yang, and Y. Liu, "A novel method of CT brain images segmentation "International Conference of Medical Image Analysis and Clinical Application, pp. 109- 112, 2010.

[12] http://www.radiologyinfo.org/‘Source for X-Ray images' 